\title{
2. SYNOPSIS
}

\begin{tabular}{|l|l|}
\hline Name of Sponsor: I.R.I.S., 50 rue Carnot - 92284 Suresnes Cedex - France & (For National \\
Authority Use only) \\
Nest drug
\end{tabular}

\section{Investigators:}

\section{Study centres:}

Two centres located in two countries included at least one patient: one centre in France included 53 patients and one centre in Spain included 51 patients.

\section{Publication (reference):}

Not applicable

\begin{tabular}{|l|l|}
\hline $\begin{array}{l}\text { Studied period: } \\
\text { Initiation date: 20 February } 2012 \text { (date of first visit first patient) } \\
\text { Completion date: 28 July 2016 (date of last withdrawal visit last patient) }\end{array}$ & $\begin{array}{l}\text { Phase of development of the } \\
\text { study: } \\
\text { Phase I }\end{array}$ \\
\hline
\end{tabular}

Completion date: 28 July 2016 (date of last withdrawal visit last patient) $\quad$ Phase I

\section{Objectives: \\ Primary objectives}

- To determine the safety of S 49076 in patients with advanced solid tumours by assessment of the Maximum Tolerated Dose (MTD), the Dose-Limiting Toxicities (DLT) assessed during cycle 1 and the adverse event profile (assessed at safety visits).

- To establish the Recommended Dose (RD) and select the S 49076 treatment schedule.

\section{Secondary objectives}

- To determine the pharmacokinetic (PK) profile of S 49076 and metabolites.

- To determine the pharmacodynamic (PD) profile of S 49076:

- To evaluate the biological activity of S 49076 using blood samples (mandatory) and tumour cells obtained from tumour biopsies (optional) before and after treatment.

- To assess the presence of circulating tumour cells (CTC) in the peripheral blood before and after treatment only during dose-escalation.

- To measure tumour response to the oral capsule form of S 49076 based on RECIST 1.1 (Eisenhauer et al, 2009).

Methodology:

First-in-human, international, multicentre, non-randomised, non-comparative, dose-escalation, open phase I study.

This study was performed in strict accordance with Good Clinical Practice including the archiving of essential documents.

\section{Number of patients:}

Planned:

- In the dose-escalation phase: not determined before start of the study due to dose-escalation procedure.

- In the expansion phase: up to 30 patients at the identified RD of the selected treatment schedule.

Included: 104 patients ( 80 patients in the dose-escalation phase and 24 additional patients in the expansion phase, i.e., 28 patients treated at the RD) 


\section{Diagnosis and main criteria for inclusion:}

Male or female patient aged $\geq 18$ years with histologically confirmed diagnosis of advanced solid tumours, with measurable or evaluable disease as defined by RECIST 1.1, that had relapsed or was refractory to standard therapy or for which no effective standard therapy was available. Estimated life expectancy $>12$ weeks. Eastern Cooperative Oncology Group (ECOG) performance status $\leq 1$. Adequate haematological, renal and hepatic functions.

\section{Test drug:}

S 49076: $7.5 \mathrm{mg}, 30 \mathrm{mg}$ and $100 \mathrm{mg}$ hard gelatine capsules (those of $100 \mathrm{mg}$ added by Amendment No 4), oral administration, taken once daily i.e. qd (arm QD) or twice daily i.e. bid (arm BID), 12 hours apart, without food (at least 30 min prior to, or at least 2 hours after a meal).

\section{Dose-escalation}

This study consisted of dose-escalation in two independent arms in order to investigate two dosage regimens QD and BID. The dose-escalation followed an algorithm-based " $3+3$ " design as follows:

- If no S 49076-related AE grade $\geq 2$ was observed during cycle 1, subsequent dose increments were $100 \%$.

- If an S 49076-related AE grade 2 was observed during cycle 1, subsequent dose increments were $50 \%$.

- If an S 49076-related AE grade $\geq 3$ was observed during cycle 1, subsequent dose increments were $25 \%$.

Three patients who met the eligibility criteria were enrolled at the initial dose level in arm QD (15 mg qd), and three other patients were enrolled at the initial dose level in arm BID (7.5 $\mathrm{mg} \mathrm{bid})$ in the schedule 1 as follows:

- Arm QD: patients received orally the capsule(s) of S 49076 once per day (QD), on a continuous dosing schedule, during a 21-day cycle ("Schedule 1" QD").

- Arm BID: patients received orally the capsule(s) of S 49076 twice per day (BID), 12 hours apart, on a continuous dosing schedule, during a 21-day cycle ("Schedule 1 BID").

In each arm, the 3 patients were observed for acute toxicity by assessment of DLT (see below) during cycle 1:

- If no DLT was observed for any of the three patients, the next dose level was explored.

- If a DLT was observed for just one of the three patients, up to three additional patients were added at this dose level. If no additional DLT was observed, the next dose level was explored.

The dose-escalation continued until at least two patients among a dose-cohort of 3 to 6 patients experienced DLT. The MTD was defined at that dose level.

DLT assessment:

DLT was determined for each patient after the completion of cycle 1. Toxicities were graded according to the Common Terminology Criteria for Adverse Events (CTCAE) version 4.0.

Assessment of the RD and selection of the S 49076 treatment schedule:

RD assessment:

The RD could be defined as one dose step below MTD or could be a dose inferior to MTD based on the safety and $\mathrm{PK} / \mathrm{PD}$ results obtained during the dose-escalation.

Selection of the $S 49076$ treatment schedule:

At the end of dose-escalation with schedule 1, once the MTD of schedule 1 was defined in each arm (MTD1 QD, MTD1 BID), a meeting between the sponsor, and the investigators took place to decide jointly the treatment schedule (among 3) to be initiated according to the safety and PK/PD results obtained during schedule 1:

- Option 1: continuation of schedule 1. The dose-escalation was to be stopped and one of the dosage regimens, either QD or BID, was selected and evaluated at the expansion cohort at the RD1.

- Option 2: initiation of continued in both arms. Subsequent cohorts of patients received S 49076 BID, until MTD2 of schedule 2 in each arm (QD and BID) was reached.

(schedule 2). The dose-escalation was to be Option 3: initiation of (schedule 3). The dose-escalation was to be continued in both arms. Subsequent cohorts of patients received S 49076 either QD or BID, until MTD3 of schedule 3 in each arm (QD and BID) was reached

Once the MTD of the tested schedule was defined in each arm, a meeting between the sponsor and the investigators took place to decide jointly either to maintain the tested schedule for expansion cohort or to initiate the remaining schedule not tested, according to the safety and PK/PD results obtained. 


\section{Expansion cohort}

Up to 30 additional patients were planned to be included and treated at the RD of the selected dosage regimen and treatment schedule to better evaluate cumulative toxicity if any.

Batch numbers: 7.5 mg: L0045230; 30 mg: L0043942, L0049126, L0049908, L0051692; 100 mg: L0053145, L0054565, L0055494, L0057029.

Comparator (Reference product and/or placebo): None

Duration of treatment:

Each patient received at least 2 cycles of treatment if DLT was not reached.

Each patient received the treatment(s) as long as, in the investigator's opinion, they received benefit according to tumour evaluation every 2 cycles. The maximum number of cycles was at the discretion of the investigator.

\section{Criteria for evaluation:}

Efficacy measurements:

Tumour evaluation based on revised RECIST was performed at baseline within 14 weeks before the first S 49076 administration and on week 3 of cycle 2 (between D15 and D21), then every 2 cycles and at the withdrawal visit (this last tumour evaluation is at the investigator's discretion).

\section{Safety measurements:}

Determination of the MTD, DLTs (assessed during cycle 1) and the adverse event profile of S 49076 were the primary objectives as well as RD establishment and selection of the S 49076 treatment schedule.

The safety criteria were DLTs, adverse events, clinical laboratory evaluation (biochemistry, haematology and coagulation), vital signs (ECOG, weight, SBP, DBP, HR), 12-lead ECG (central reading), LVEF.

\section{Pharmacokinetic measurements}

Blood and urinary samples were collected as well as tumour biopsies (at same time-points as for biomarkers). See appended PK reports.

Drug-drug interaction and endogenous metabolites (including 4beta-hydroxycholesterol) See separate and appended bioanalytical reports, respectively.

\section{Pharmacodynamic measurements (biomarkers)}

Tumour biopsies for biomarkers measurements were optional and collected within 14 days prior to the first administration of S 49076 and between 2 and 8 hours after S 49076 administration on week 2 (between D8 and D14) of cycle 1 or cycle 2. Blood samples were mandatory (except for patients included before approval of Amendment No. 4) and collected for genomic and proteomic measurements at the same time-points as those for tumour biopsies. Presence of Circulating Tumour Cells (CTC) was measured in the peripheral blood of patients (only during dose-escalation). 


\section{Statistical methods: \\ Analysis Set:}

- Screened Set: all screened patients.

- Included Set (IS): all included patients.

- Safety Set (SS): all patients having taken at least one dose of study treatment.

- Full Analysis Set (FAS): based on the intention-to-treat principle and, all included patients who had taken at least one dose of study treatment and without any relevant deviation at baseline.

- Response Evaluable Set (RES): all patients in the FAS who had at least one baseline and one post-baseline tumour evaluation.

- DLT Evaluable Set (DLTES): all patients of the SS who were evaluable for DLT according to the DLT assessment at end of cycle 1 . A patient was not considered evaluable if:

- $\mathrm{He} / \mathrm{she}$ discontinued during cycle 1 for reason other than DLT or

- $\mathrm{He} / \mathrm{she}$ did not undergo a DLT assessment at the start of cycle 2 or

- $\mathrm{He} / \mathrm{she}$ did not receive at least $85 \%$ of S 49076 doses, unless treatment was stopped for a DLT.

\section{Efficacy analysis:}

Efficacy analyses were done in the FAS and RES. The Best Overall Response (BOR) was provided by dose level, arm (QD/BID) and overall (Arm QD + Arm BID), whereas the Objective Response Rate (ORR) and the Clinical Benefit Rate (CBR) as well as the duration of Clinical Benefit, the Progression Free Survival (PFS) and the Overall Survival (OS) were provided in tables and/or graphs by arm, on the RD and overall. All dose levels were presented in listings. The overall response (OR) was evaluated according to the investigator assessments. The survival functions of the time dependent parameters (duration of Clinical Benefit, OS and the PFS) were estimated via Kaplan-Meier curves.

Others efficacy analysis were relative change from (baseline/smallest) of the sum of the lesions diameters and best relative change from baseline of the sum of the lesions diameters.

\section{Study outcome}

Disposition of patients (status, protocol deviations and analysis set), initial tumour assessment as well as treatment duration and extent of exposure were described by dose level, by arm and overall while all other baseline characteristics and concomitant treatments were described by arm, on the RD and overall.

\section{Safety analysis}

The safety analysis was assessed with a description of the DLTs in the DLTES and of emergent adverse events (EAEs) and serious AEs (SAEs), death, biology parameters, vital signs, ECG and LVEF in patients of the SS by dose level and overall.

\section{Pharmacodynamic analyses (biomarkers)}

Circulating proteins: description at each visit using available value and relative changes of interest, global evolution and evolution according to class of total daily dose in FAS as well as evolution according to response group $(\mathrm{CBR}=\mathrm{Yes} / \mathrm{No})$ in FAS and RES, by arm and overall.

IHC biomarkers: percentage of patients with Hscore or Intensityl $>10$ in IS, description at baseline of all markers in FAS, individual evolution by class of total daily dose by arm (only for phosphorylated markers) in FAS; description according to response group using available value at baseline, in RES and FAS. .

FISH biomarkers: percentage of patients in each amplification class at baseline in the IS and according to response group in RES and FAS. Description of biomarkers with amplification in at least 2 patients in FAS.

CGH biomarkers: percentage of patients in each CGH status at baseline in IS and according to response group in RES and FAS. A comparison between FISH and CGH technologies was performed.

Circulating Tumor Cells (CTC): percentage of patients with positive CTC for at least one time point, CTC evolution by visit with a graph according to BOR as well as correlation between CTC at baseline and PFS on patients with adenocarcinoma of colon by arm and overall in FAS and RES.

Pharmacokinetic analysis: see separated PK report. 


\section{SUMMARY - CONCLUSIONS \\ DISPOSITION OF PATIENTS AND ANALYSIS SETS}

A total of 132 patients were screened for the study and 104 patients were included. All patients were withdrawn for the following reasons: 87 patients (84\%) for progressive disease, 15 patients (14\%) for adverse event, and 2 patients for non-medical reason (including one patient non-treated).

One included patient was still on-going in the follow-up period at the time of the final analysis. Data not available will be presented in an addendum to this clinical study report.

Number of patients in each analysis sets are presented in Table 1.

Table 1 - Analysis sets

\begin{tabular}{lccccc}
\hline Analysis sets & & Arm QD & Arm BID & Not treated & All \\
\hline Screened Set & $\mathrm{n}$ & 68 & 35 & 29 & 132 \\
Included Set & $\mathrm{n}$ & 68 & 35 & 1 & 104 \\
$\quad$ Safety Set & $\mathrm{n}(\%)$ & $68(100)$ & $35(100)$ & - & $103(99.0)$ \\
$\quad \mathrm{nLT}(\%)^{1}$ & $63(92.6)$ & $34(97.1)$ & - & $97(94.2)$ \\
$\quad \mathrm{n}(\%)$ & $68(100)$ & $35(100)$ & - & $103(99.0)$ \\
$\quad$ Full Analysis Set & $\mathrm{n}(\%)^{2}$ & $60(88.2)$ & $32(91.4)$ & - & $92(89.3)$ \\
$\quad$ Response Evaluable Set & n: number of patient in each arm concerned; \%: calculated as \% of the IS in each arm concerned. \\
(1): calculated as \% of the SS in each arm concerned; (2): calculated as \% of the FAS in each arm concerned
\end{tabular}

\section{STUDY POPULATION AND OUTCOME}

\section{Main baseline characteristics}

The included patients had a median age of 60.0 years old (mean $=58.3 \pm 10.8$ years), and the majority were below 65 years old (63.5\% overall). More than half of the patients were male $(58.7 \%$ overall). Demographic characteristics of patients at RD $600 \mathrm{mg} q d$ (median age $=62.5$ years, $64.3 \%$ of men) were comparable to those of overall patients.

At initial diagnosis, the primary tumour was in advanced stage for a majority of patients according to TNM classification $(\mathrm{n}=70)$ : staged T3 $=32.9 \%$ and $\mathrm{T} 4=31.4 \%$. The lymph nodes were staged N1 for $21.4 \%$ of patients, N2 for $28.6 \%$ and N3 for $4.3 \%$. Metastases were reported for $54.3 \%$ of patients.

At inclusion in the study, overall patients had an advanced solid tumour for $3.2 \pm 2.4$ years in average (median $=2.4$ years). The most frequent tumour were adenocarcinoma of colon in 17 patients $(16.4 \%)$, choroid melanoma in 11 patients $(10.6 \%)$, lung adenocarcinoma and pleural mesothelioma malignant, each of these in 8 patients $(7.7 \%)$. Most of patients $(97.1 \%)$ had metastases.

Patients started their last treatment line prior to the relapse for $3.7 \pm 4.4$ months in average (median $=2.7$ months) before inclusion. The last treatment line was terminated for an average of $105.5 \pm 99.7$ days (median $=77$ days) before the first S 49076 intake; this interval was longer in the arm BID (median $=91$ days) than in the arm QD (median $=69$ days). The mean duration between relapse and the inclusion visit was $2.2 \pm 1.8$ months (mean $=1.8$ months). The majority of patients had received three $(19.2 \%)$ or more than three $(53.9 \%)$ lines of prior drug treatments.

At baseline, 100 patients $(96.2 \%)$ had measurable target lesions.

The mean weight was $71.8 \pm 15.4 \mathrm{~kg}$, and ECOG performance status was $\leq 1$ for all patients. Mean SBP, DBP, and heart rate were $117.5 \pm 17.2 \mathrm{mmHg}, 71.6 \pm 11.3 \mathrm{mmHg}$, and $79.9 \pm 14.2 \mathrm{bpm}$, respectively. Regarding ECG, the mean QTcB interval was $414.5 \pm 20.1 \mathrm{~ms}$. The mean LVEF was $62.7 \pm 4.4 \%$, ranging from $55 \%$ and $77 \%$.

In summary, the main baseline characteristics were comparable between arm QD and BID, except for duration since last prior line of treatment intake prior to the relapse which was longer in arm BID (median $=3.5$ months) than in arm QD (median $=2.5$ months).

\section{Extent of exposure}

In the Full Analysis Set $(\mathrm{N}=103$, Safety Set identical to FAS), the mean S 49076 treatment duration was $11.9 \pm 15.6$ weeks $($ median $=6.1$ weeks), ranging from 1 day to 100 weeks. The median number of cycles received was 2.0 cycles. The mean relative dose intensity per patient (i.e. compliance) was $95.0 \pm 11.1 \%$ (median $=99.6 \%$ ) ranging from 52.4 to $130 \%$. The median compliance was $99.2 \%$ for patients at RD $600 \mathrm{mg} q d$. The median cumulative dose received by patient was $14535 \mathrm{mg}$.

This Phase I study was conducted in patients with advanced solid tumours, for which baseline characteristics were in line with the target population defined in the study protocol. 


\section{SUMMARY - CONCLUSIONS (Cont'd)}

\section{EFFICACY RESULTS}

Overall in the RES (N $=92)$, the BOR was Stable Disease (SD) in 46 patients $(50.0 \%): 27$ patients $(45.0 \%)$ in the arm QD, 19 patients (59.4\%) in the arm BID and 12 patients (44.4\%) at RD $600 \mathrm{mg} q d$. Progressive Disease (PD) was observed in 40 patients (43.8\%): 30 patients $(50.0 \%)$ in the arm QD, 10 patients $(31.3 \%)$ in the arm BID and 12 patients (44.4\%) at RD $600 \mathrm{mg} q d$. Otherwise, the BOR was Partial Response (PR) in 1 patient (not confirmed following initial documentation), Non-Complete Response (CR)/Non-PD in 3 patients and not evaluable (NE) in 2 patients.

In the RES, an objective response (not confirmed) was observed in a single patient. The overall clinical benefit rate (CBR) was $22.8 \%$ (21 patients); it was $16.7 \%$ (10 patients) in the arm QD, $18.5 \%$ (5 patients) at RD and $34.4 \%$ (11 patients) in the arm BID.

The overall median duration of the clinical benefit was 26.1 weeks while longer duration was observed in arm $\mathrm{QD}$ (median $=39.1$ weeks) and at RD (median $=33.5$ weeks). The overall median PFS was 7.3 weeks while longer duration was observed in arm BID (median $=12.0$ weeks).

Regarding circulating biomarker assessments, significant increases of serum HGF $(p=0.001)$ and FGF23 $(\mathrm{p}<0.001)$ were detected at cycle 1 compared to baseline without a dose effect. Changes in HGF and FGF23 were not associated with the clinical response. Regarding protein biomarkers assessed by IHC, two patients highly expressed AXL (H score > 150) at baseline. Three patients were positive for phospho-MET at baseline and no more after treatment. For pan-phospho-Tyrosine a small decrease tendency at cycle 1 and cycle 2 was observed. No particular protein biomarker profile was identified at baseline as predictive of response (very few patients with IHC data in each response group). Regarding genomic biomarkers assessed by FISH or CGH, FGFR1 was amplified in 2 patients who were not responders. Regarding circulating tumour cells (CTC) in patients with colon adenocarcinoma $(n=14), 13$ patients had at least one positive CTC value but no interpretation of the results could be due to low number of patients.

\section{SAFETY RESULTS}

\section{Dose-escalation: $M T D, R D$ and schedule finding}

Overall, 103 patients received at least one dose of S 49076 on a 21-day cycle continuous dosing schedule from $15 \mathrm{mg}$ to $900 \mathrm{mg}$ total daily dose. At the end of cycle 1,97 patients were evaluable for DLT.

In the QD arm (dose-escalation), S 49076 was escalated through 11 dose levels in 44 patients: $15 \mathrm{mg} q d$ $(\mathrm{n}=3), 22.5 \mathrm{mg} q d(\mathrm{n}=3), 30 \mathrm{mg} q d(\mathrm{n}=6), 45 \mathrm{mg} q d(\mathrm{n}=3), 67.5 \mathrm{mg} q d(\mathrm{n}=4), 180 \mathrm{mg} q d(\mathrm{n}=3), 270 \mathrm{mg}$ $q d(\mathrm{n}=6), 405 \mathrm{mg} q d(\mathrm{n}=3), 600 \mathrm{mg} q d(\mathrm{n}=4), 760 \mathrm{mg} q d(\mathrm{n}=6), 900 \mathrm{mg} q d(\mathrm{n}=3)$. Six DLTs were observed in 5 patients. The first DLT was observed at $30 \mathrm{mg} q d$ (grade 2 LVEF decreased), 3 further DLTs were observed at $900 \mathrm{mg}$ (grade 4 neutropenia and thrombocytopenia both in one patient, and grade 1 dizziness in one patient). Considering no DLT was observed at the previous dose $600 \mathrm{mg} q d$, it was decided to investigate an intermediate dose $760 \mathrm{mg} q d$. Two patients had DLT (grade 2 LVEF decreased) at $760 \mathrm{mg} q d$ which was defined as the MTD. The RD was defined as $600 \mathrm{mg} q d$.

In the BID arm (dose-escalation), S 49076 was escalated through 8 dose levels in 35 patients: $7.5 \mathrm{mg}$ bid $(\mathrm{n}=3), 15 \mathrm{mg}$ bid $(\mathrm{n}=3), 30 \mathrm{mg}$ bid $(\mathrm{n}=3), 60 \mathrm{mg}$ bid $(\mathrm{n}=3), 120 \mathrm{mg}$ bid $(\mathrm{n}=3), 180 \mathrm{mg}$ bid $(\mathrm{n}=7)$, $225 \mathrm{mg}$ bid $(\mathrm{n}=6), 285 \mathrm{mg}$ bid $(\mathrm{n}=7)$. Four DLTs were observed in 4 patients: grade 3 hypotension at $180 \mathrm{mg}$ bid, grade 2 LVEF decreased at $225 \mathrm{mg}$ bid, grade 3 hallucination/cerebellar syndrome and grade 2 LVEF decreased at $285 \mathrm{mg}$ bid which was defined as the MTD.

The global safety profile was comparable in both arms and PK data showed no accumulation of S49076 with lower ratio in $q d$ than bid administration. Based on these safety and PK results, the once daily dosing was chosen.

Twenty four patients $(n=24)$ were then included in the expansion cohort and received S 49076 at $\mathrm{RD} 600 \mathrm{mg} q d$. The safety data in overall patients treated at $\mathrm{RD}$ (during dose-escalation and expansion parts, $\mathrm{n}=28$ ) confirmed the RD and the treatment schedule.

None of the two others intermittent dosing schedules was investigated. 


\section{SUMMARY - CONCLUSIONS (Cont'd) \\ SAFETY RESULTS (Cont'd) \\ Emergent adverse events}

Main results for adverse events in the SS are described in the Table 2.

Table 2 - Overall summary for adverse events in the Safety Set

\begin{tabular}{|c|c|c|}
\hline & & $\begin{array}{c}\text { All } \\
(N=103)\end{array}$ \\
\hline \multicolumn{3}{|l|}{ Patients having reported } \\
\hline at least one EAE & $\mathrm{n}(\%)$ & $101(98.1)$ \\
\hline at least one treatment-related EAE & $\mathrm{n}(\%)$ & $83(80.6)$ \\
\hline at least one severe EAE & $\mathrm{n}(\%)$ & $51(49.5)$ \\
\hline \multicolumn{3}{|l|}{ Patients having experienced } \\
\hline at least one serious AE (including death) & $\mathrm{n}(\%)$ & $56(54.4)$ \\
\hline at least one serious EAE (including death) & $\mathrm{n}(\%)$ & $53(51.5)$ \\
\hline at least one treatment-related serious EAE & $\mathrm{n}(\%)$ & $11(10.7)$ \\
\hline \multicolumn{3}{|l|}{ Patients with treatment withdrawal } \\
\hline due to an EAE & $\mathrm{n}(\%)$ & $22(21.3)$ \\
\hline due to a treatment-related EAE & $\mathrm{n}(\%)$ & $10(9.7)$ \\
\hline due to a serious EAE & $\mathrm{n}(\%)$ & $14(13.6)$ \\
\hline due a treatment-related serious EAE & $\mathrm{n}(\%)$ & $4(3.9)$ \\
\hline \multicolumn{3}{|l|}{ Patients who died } \\
\hline during the treatment period & $\mathrm{n}(\%)$ & $9(8.7)$ \\
\hline during the follow-up period* & $\mathrm{n}(\%)$ & $53(51.5)$ \\
\hline
\end{tabular}

During the study, 101 patients $(98.1 \%)$ reported at least one EAE. The affected SOCs in more than half of the patients were general disorders and administration site conditions (71\%), gastrointestinal disorders (64\%), nervous system disorders (55\%) and metabolism and nutrition disorders (52\%).

Overall, the most frequently reported EAEs were peripheral oedema (49\%), hypoalbuminemia (37\%) and asthenia (33\%). Most EAEs (75\% of EAEs) recovered at the time of the report. No relevant difference was observed between arms QD and BID except vomiting and dry mouth more frequent in arm QD $(21 \%$ and $19 \%$, respectively) than in arm BID (11\% and 9\%, respectively). At RD $600 \mathrm{mg} q d$, the most frequent EAEs were peripheral oedema (64\%), hypoalbuminemia (50\%), yellow skin (39\%), dysaesthesia (36\%) and dry mouth $(32 \%)$ : all of them were more frequently reported than in overall patients.

Overall, 36 patients (35\%) had at least one Grade 3 EAE; 3 patients at least one Grade 4 EAE and 12 patients had fatal EAE including 5 malignant neoplasm progression. None of the fatal EAEs was considered as related to the study treatment, except large intestine perforation.

Overall, 83 patients $(81 \%)$ reported at least one EAE considered as treatment-related by the investigator. This frequency was higher at RD $600 \mathrm{mg} q d(96 \%)$ than in overall patients. It was higher in the arm BID (89\%) than in the arm QD (76\%). The most common treatment-related EAE were peripheral oedema (35\%), hypoalbuminaemia (31\%), yellow skin $(26 \%)$ likely related to drug coloration, dysaesthesia $(21 \%)$, asthenia $(17 \%)$, dry mouth (16\%), diarrhoea (14\%) and dysgeusia (12\%). Most of them were more frequently reported at RD $600 \mathrm{mg} q d$ than in the overall patients; no relevant difference between arms QD and BID was observed except for dry mouth and diarrhoea.

Overall, 22 patients (21\%) experienced at least one EAE leading to treatment withdrawal: 9 patients $(13 \%)$ in arm QD, 13 patients $(37 \%)$ in arm BID and 6 patients $(21 \%)$ at RD 600 qd. Those reported in more than one patient were peripheral oedema $(n=2)$, pulmonary embolism $(n=2)$, metastases to central nervous system $(\mathrm{n}=2)$, LVEF decreased $(\mathrm{n}=3)$ and dyspnoea $(\mathrm{n}=3)$. In 10 patients $(10 \%)$, these EAEs were considered as treatment-related.

Overall, 56 patients (54\%) experienced 95 serious adverse event during the study including 85 serious emergent adverse events in 53 patients (51.5\%). The most frequent SEAEs were malignant neoplasm progression in 7 patients, including 3 patients at RD $600 \mathrm{mg} q d$, pleural effusion in 6 patients, including 3 patients at $\mathrm{RD}$, dyspnoea in 5 patients, including 2 patients at $\mathrm{RD}$, pulmonary embolism in 5 patients, including 2 patients at RD, and lung infection in 3 patients, including 1 patient at RD. Overall, 16 SEAEs were considered as treatment-related in 11 patients ( 5 patients in arm QD, all at RD $600 \mathrm{mg} q d$, and 6 patients in the arm BID) and 6 of those led to study drug withdrawal in 4 patients: fatal large intestine perforation (180 mg bid), cerebellar syndrome and hallucination/visual (both in one patient, $285 \mathrm{mg}$ bid) hypotension and syncope (both in one patient, $180 \mathrm{mg}$ bid) and bullous dermatitis (285 $\mathrm{mg}$ bid). 


\section{SUMMARY - CONCLUSIONS (Cont'd) \\ SAFETY RESULTS (Cont'd)}

\section{Emergent adverse events (Cont'd)}

Overall, 56 patients (54\%) experienced 95 serious adverse event during the study including 85 serious emergent adverse events in 53 patients (51.5\%). The most frequent SEAEs were malignant neoplasm progression in 7 patients, including 3 patients at RD $600 \mathrm{mg} q d$, pleural effusion in 6 patients, including 3 patients at RD, dyspnoea in 5 patients, including 2 patients at RD, pulmonary embolism in 5 patients, including 2 patients at RD, and lung infection in 3 patients, including 1 patient at RD. Overall, 16 SEAEs were considered as treatment-related in 11 patients (5 patients in arm QD, all at RD $600 \mathrm{mg} q d$, and 6 patients in the arm BID) and 6 of those led to study drug withdrawal in 4 patients: fatal large intestine perforation (180 mg bid), cerebellar syndrome and hallucination/visual (both in one patient, $285 \mathrm{mg}$ bid) hypotension and syncope (both in one patient, $180 \mathrm{mg}$ bid) and bullous dermatitis $(285 \mathrm{mg}$ bid).

Sixty-two patients $(60.2 \%)$ died during the study: 9 (8.7\%) during the treatment period and $53(51.5 \%)$ during the follow-up period.

During the treatment period: 8 patients died in the arm QD, 6 patients for disease progression, (for three of them a fatal adverse event was also reported: pleural effusion, cardiac failure, dyspnoea), 1 patient for pulmonary haemorrhage and 1 patient for bile duct obstruction (both not treatment-related); 1 patient died in the arm BID for large intestine perforation (treatment related).

During the follow-up period i.e. after the withdrawal visit, 53 patients (51.5\%) died: 39 patients for disease progression, none for toxicity caused by the study protocol, 11 patients for other reason (not specified except one disease progression) and, 3 patients for missing reason.

\section{Blood laboratory evaluation}

For the biochemistry parameters, the most frequently emergent severe abnormal values (graded $\geq 3$ ) were low phosphates ( $8 \%$ of patients), high ALP (8\%), low albumin (6\%) and high GGT (6\%). Four emergent grade 4 values were reported for low magnesium in 2 patients, high GGT and total bilirubin each in 1 patient: among those, a fatal EAE bile duct obstruction was reported for high total bilirubin. Other grade 4 values were not reported as adverse event. For non-gradable parameters, the most frequently reported emergent abnormal values (i.e. out of normal range laboratory) were low total protein $(70 \%)$ and high bicarbonate $(48 \%)$. The high percentage of patients with low total protein was consistent with a high percentage of patients $(77.5 \%)$ with low albumin obtained when taking in account severe (graded $\geq 3$ ) and non-severe (graded 1 and 2) abnormal low albumin values.

For the hematological parameters, the most frequently emergent severe abnormal values (graded $\geq 3$ ) were low lymphocytes (11\% of patients). Five emergent grade 4 values were reported: low neutrophils and WBC with EAE grade 3 febrile neutropenia in one patient; low neutrophils, low WBC and low platelets with EAEs grade 4 febrile neutropenia and thrombocytopenia, both DLTs at $900 \mathrm{mg} q d$, as well as grade 4 anemia in one patient. For the non-gradable parameters, the most frequently reported emergent abnormal values (i.e. out of normal range laboratory) were high neutrophils (33\%), low haematocrit (21\%) and low red blood cells $(20 \%)$.

For the coagulation parameters, emergent grade 3 for aPTT prolonged was reported in 4 patients, emergent out-of-range values for low aPTT and low PT, each in 7 patients. For these parameters, no EAE graded $\geq 3$ was reported.

\section{Other safety evaluation}

The majority of patients (62.4\%) had ECOG PS score 1 under treatment, including $22.8 \%$ of patients with score 0 at baseline. The weight of patients was stable during the treatment period.

Regarding blood pressures, the mean change from baseline to highest SBP and DBP values were $8.9 \pm 14.9 \mathrm{mmHg}$ (median $=10 \mathrm{mmHg}$ ) and $9.0 \pm 10.3 \mathrm{mmHg}$ (median $=8.0 \mathrm{mmHg}$ ), respectively. The mean change from baseline to lowest SBP and DBP values were $-18.0 \pm 14.7 \mathrm{mmHg}$ (median $=-17 \mathrm{mmHg}$ ) and $-8.1 \pm 10.1 \mathrm{mmHg}$ (median $=-7 \mathrm{mmHg}$ ), respectively.

The mean change in heart rate from baseline to lowest value was $-5.4 \pm 11.7 \mathrm{bpm}$ (median $=-5.0 \mathrm{bpm}$ ) and was $15.3 \pm 12.1 \mathrm{bpm}($ median $=16.0)$ from baseline to highest value.

A total of 25 patients $(24 \%)$ had at least one emergent ECG abnormality that was considered as clinically significant according to central reading for 3 patients: relevant QTcF prolongation $>480$ msec and first degree atrio-ventricular block in 1 patient, Brugada pattern in 1 patient, both ventricular and atrial isolated premature beat in 1 patient. Regarding QTcB on treatment, 10 patients $(10 \%$ of overall $)$ had QTcB $>450 \mathrm{~ms}$ as following: ]450; 480] $\mathrm{ms}(\mathrm{n}=8)] 480 ; 500,] \mathrm{ms}(\mathrm{n}=1)$ and $>500 \mathrm{~ms}(\mathrm{n}=1)$. Change of QTcB from baseline was ] $30 ; 60] \mathrm{ms}$ in 15 patients $(14.6 \%)$ and $>60 \mathrm{~ms}$ in 3 patients $(2.9 \%)$. Overall, the investigator reported ECG QT prolonged (QTcB values of 466 and $509 \mathrm{~ms}$ ) as EAE in two patients: one EAE serious, one treatmentrelated, both recovered. 


\section{SUMMARY - CONCLUSIONS (Cont'd)}

SAFETY RESULTS (Cont'd)

\section{Other safety evaluation (Cont'd)}

LVEF assessment was performed in 96 patients on treatment. The decrease of LVEF value from baseline was greater or equal to $10 \%$ in 13 patients. Ten 'LVEF decreased' were reported as EAE (10 patients, 10\%), all treatment-related. All, except one, were grade 2, recovered or recovering, without S 49076 dose relationship, and confounding factors were present in most cases.

\section{CONCLUSION}

In this first-in-human open dose-escalation phase I study with oral doses of $S 49076$, a total of 103 patients with advanced solid tumours were included and treated either once daily $(q d)$ in 68 patients or twice daily (bid) in 35 patients in continuous 21-day cycles. The $q d$ maximum tolerated dose (MTD) was reached at $760 \mathrm{mg}$ and the bid MTD was $285 \mathrm{mg}$. The recommended dose (RD) was determined at $600 \mathrm{mg} q d$. Overall, 28 patients were treated at $\mathrm{RD}$, including 24 patients in the expansion cohort. The schedule continuous 21-day cycle at RD $600 \mathrm{mg} q d$ was confirmed as the optimal administration schedule.

Response to treatment was evaluable in 92 patients. The best overall response was stable disease in $50 \%$ of patients and the overall clinical benefit rate was $22.8 \%$.

The most frequent emergent adverse events considered as treatment-related by the investigator were peripheral oedema, hypoalbuminemia, yellow skin, dysaesthesia, asthenia and dry mouth.

Date of the report: 19 May 2017

Version of the report: Final Version 